\title{
Analysis of the Value of the Blood Urea Nitrogen to Albumin Ratio as a Predictor of Mortality in Patients with Sepsis
}

\author{
Tianyong Han',*, Tao Cheng',*, Ye Liao², Shiyuan Tang', Bofu Liu', Yarong He', Zhihan Gu', \\ Chenxi Lei', Yuling $\mathrm{Cao}^{3}$, Yu Cao' \\ 'Emergency Department, West China Hospital of Sichuan University, Chengdu, Sichuan, People's Republic of China; ${ }^{2}$ Medical Intensive Care Unit, \\ West China Hospital of Sichuan University, Chengdu, Sichuan, People's Republic of China; ${ }^{3}$ Operations Management Department, West China \\ Hospital of Sichuan University, Chengdu, Sichuan, People's Republic of China \\ *These authors contributed equally to this work
}

Correspondence: Yu Cao, Emergency Department, West China Hospital of Sichuan University, 37 Guoxue Lane, Wuhou District, Chengdu, 6I004I, Sichuan, People's Republic of China, Tel +028-85422288, Email caoyu@wchscu.cn

\begin{abstract}
Purpose: At present, simple, accurate, and efficient prognostic tools for the evaluation of cases with early-stage sepsis in the emergency department (ED) are lacking. An increased blood urea nitrogen to albumin ratio (BAR) has previously been shown to be a valuable biomarker with predictive utility in several diseases. The relationship between BAR and sepsis patient outcomes, however, is not well-understood. This exploration was thus developed for the exploration of the link between BAR values and the short-term prognosis of cases with sepsis.

Methods: This was a retrospective cohort research of sepsis cases admitted to the West China Hospital of Sichuan University ED from July 2015 to June 2016. Laboratory data were collected upon ED admission, and 7-day all-cause mortality was the primary study endpoint. Relationships between BAR values and APACE II and SOFA scores were generated assessed with correlation coefficient heatmaps. Independent risk factors were identified through multivariate analyses, with the curves of receiver operating characteristic (ROC) being employed to gauge the value of BAR as a predictor of the risk of mortality in sepsis cases.

Results: In sum, 801 patients participated in the present investigation. BAR values were strongly correlated with APACHE II and SOFA scores. In a multivariate logistic regression assessment, BAR was identified as an independent predictor of mortality among patients with sepsis (HR=1.032, 95\% CI: $1.010-1.055, P=0.004)$. BAR exhibited an AUC of $0.741(95 \%$ CI: $0.688-0.793, P<0.001)$ when used to predict patient mortality risk, with 5.27 being the optimal BAR cut-off.

Conclusion: We found that BAR can be used as a reliable biomarker to predict mortality in patients with sepsis.

Keywords: sepsis, blood urea nitrogen-to-albumin ratio, risk factors, prognosis
\end{abstract}

\section{Introduction}

Sepsis is one of the pervasive reasons for mortality all over the world, imposing substantial medical and economic burdens on affected individuals and society as a whole. ${ }^{1-3}$ The 2016 international consensus for sepsis and septic shock (Sepsis-3) benchmark recently defined sepsis as a form of life-threatening organ dysfunction arising as a result of abnormal responses to infection. ${ }^{4}$ Effectively identifying patients at a high risk of severe sepsis during the early stages of this disease is thus critically important as it has the potential to lower rates of multiple organ failure and associated patient mortality.

Multiple systems have been developed to gauge the severity of sepsis and to approximate the prognosis of affected patients, ${ }^{5}$ comprising the sequential organ failure assessment (SOFA) score and Acute Physiology and Chronic Health Evaluation (APACHE) II score, both of which are used to identify patients with a bad prognosis. ${ }^{6,7}$ Both of these scores, however, are dependent on many whole-body physiologic parameters and are thus challenging to measure, limiting their use 
during the initial stages of patient evaluation in the Emergency Department (ED). ${ }^{8,9}$ As such, there is a clear need to identify reliable biomarkers that can be readily assessed to predict the sepsis patient prognosis during the early stages of disease.

A growing body of evidence suggests that elevations in levels of blood urea nitrogen (BUN) and decreases in serum albumin levels are closely associated with sepsis severity. Moreover, an increase in the BUN to albumin ratio (BAR) has been confirmed to be a robust predictor of outcomes for cases with a range of diseases, such as chronic obstructive pulmonary disease (COPD), COVID-19, and critical illnesses. ${ }^{10-13}$ The role of BAR as a prognostic factor for sepsis patients, however, has not been assessed in prior reports. Here, we therefore sought to evaluate the value of BAR as a predictor of mortality risk among sepsis patients.

\section{Materials and Methods Study Design}

This was a single-center retrospective cohort research developed for the assessment of the relationship between BAR levels and the odds of negative achievements in sepsis cases. All patients or their legal proxies provided informed consent for study participation prior to enrollment. The public and patients were not included in designing, conducting, reporting, or disseminating research plans. This study was consistent with the Declaration of Helsinki, and was confirmed through the Human Ethical Committee of West China Hospital of Sichuan University (number: 2019 (373)).

\section{Study Population}

Data used for the present exploration were obtained from the West China Hospital retrospective sepsis database. Specifically, data were obtained for cases with sepsis evaluated in the ED of West China Hospital of Sichuan University between July 2015 and June 2016. Patients eligible for inclusion in this analysis were individuals $\geq 18$ years old that met the Sepsis-3 definition and were admitted within 3 days following disease onset. ${ }^{4}$ Contributors were excluded from this research if they were pregnant, exhibited malignant tumors, presented to the ED in cardiac arrest necessitating immediate cardiopulmonary resuscitation, required long-term hemodialysis, exhibited chronic kidney disease or exhibited incomplete data.

\section{Data Collection}

Data collected for patients included in this study included demographic details, medical history, vital signs, laboratory findings, and associated diagnosis. Analyzed laboratory parameters included $\mathrm{pH}$, lactate (Lac), base excess (BE), hemoglobin (Hb), platelet (PLT) count, white blood cell (WBC) count, bilirubin, hematocrit (HCT), albumin (ALB), creatinine $(\mathrm{Cr})$, blood urea nitrogen (BUN), triglyceride (TG), aspartate aminotransferase (ALT), alanine aminotransferase (AST), prothrombin time (PT), and activated partial thromboplastin time (APTT) values. BAR was calculated by dividing the BUN of a patient at admission by their ALB level. SOFA and APACHE II scores for patients were estimated by physicians based on baseline data following ED admission.

\section{Endpoints and Follow-Up}

The patients received a structured telephone interview-based follow-up call at 28 days post-ED admission. The primary study endpoint was 7-day all-cause mortality, while secondary endpoints included mechanical ventilation (MV), the incidence of ICU admission (AICU), and 28-day mortality. AICU was described as patient admission to the ICU owing to unstable vital signs, severe hydroelectrolyte imbalance, severe dystrophy, and a requirement for organ support. MV was described as a requirement for invasive or non-invasive ventilation as a consequence of hypoxic respiratory failure. ${ }^{14}$ Patients were separated into survival and non-survival groups based on their 7-day survival status.

\section{Statistical Analysis}

The Kolmogorov-Smirnov assessment was employed to evaluate whether continuous variables conformed to a normal distribution, with those that did being expressed as means \pm standard deviations and scrutinized via Student's t-tests. Nonnormally distributed continuous variables were presented as medians [interquartile range (IQR)] and were scrutinized via 
Mann-Whitney $U$-tests. Non-continuous variables were presented as frequencies (proportions) and scrutinized employing $\chi^{2}$ or Fisher's exact test as appropriate. Relationships between BAR values, APACHE II scores, and SOFA scores in patients with sepsis were assessed using a correlation coefficient heat map, with the Pearson's correlation test being used to define correlation coefficients. The utility of BAR as an independent predictor of short-term mortality in sepsis patients was assessed through an analysis of multivariate logistic regression. The area under the receiver operating characteristic (ROC) curve (AUC) for BAR, APACHE II, and SOFA scores was calculated to gauge the ability of these different metrics to predict the odds of 7-day mortality in septicemic cases. The AUC values for these variables were compared via the DeLong test. The maximum Youden index was utilized to select an optimal BAR cut-off value for stratifying patients into two subgroups, with rates of adverse events being compared between these groups (including AICU, MV, and 28day mortality) via Fisher's exact test or $\chi^{2}$ test. A two-sided $\mathrm{P}<0.05$ was the threshold of significance, and all outcomes were evaluated employing SPSS 26.0 (IBM Corporation, NY, USA), MedCalc Statistical Software v 19, and R V.3.5.1 (R Foundation for Statistical Computing, Vienna, Austria).

\section{Results}

\section{Baseline Characteristics}

In sum, 801 sepsis cases participated in this survey (mean age: $57.37 \pm 17.73$ years old; $64.4 \%$ male). These patients exhibited respective median SOFA and APACHE II scores of 5 (IQR 3-8) and 15 (IRQ 11-20). Of these cases, 722 (90.14\%) survived for 7 days, while 79 (9.86\%) died during this period. Clinical data were compared between these two groups of patients, revealing significant differences in heart rate, respiratory rate, mean arterial pressure, $\mathrm{SpO}_{2}, \mathrm{PH}$, lactate, base excess, platelet count, aspartate aminotransferase, serum albumin, BUN, BAR, creatinine, activated partial thromboplastin time, prothrombin time, SOFA scores, and APACHE II scores between groups $(P<0.05$, Table 1). No other clinical characteristics were found to vary between these groups $(P>0.05$, Table 1$)$.

\section{Correlations Between BAR Values and SOFA and APACHE II Scores}

Next, we explore the relationship between BAR values and both the APACHE II scores and SOFA scores for sepsis patients through Pearson's correlation analyses. This approach revealed that BAR values and SOFA scores were associated n ( $\mathrm{r}=0.47, P<0.001$, Figure 1), with a similarly strong positive correlation being observed between BAR values and APACHE II scores (r=0.34, $P<0.001$, Figure 1).

\section{Evaluation of the Diagnostic Performance of BAR Values as Predictors of the Odds of 7-Day Mortality Among Sepsis Patients}

Next, we examined the utility of BAR, APACHE II, and SOFA scores as predictors of 7-day mortality among sepsis patients using ROC curves. The AUC values for these curves indicated that BAR values exhibited good predictive utility (AUC $=0.741,95 \%$ CI: $0.688-0.793, P<0.001)$ that was not inferior to SOFA $(0.772,95 \%$ CI $0.732-0.813)$ or APACHE II $(0.772,95 \%$ CI $0.732-0.813)$ performance, with no significant differences among these indicators $(P>0.05)$ (Figure 2$)$. The optimal BAR value for the estimation of 7-day mortality among sepsis patients was 5.27 , yielding respective sensitivity and specificity values of $86.08 \%$ and $50.00 \%$.

\section{The Relationship Between BAR Values and Adverse Outcomes}

Septic patients were next classified into groups with high $B A R$ values $(B A R \geq 5.27)$ and low $B A R$ values $(B A R<5.27)$ based on the optimal BAR cut-off value. Rates of adverse events including AICU, MV, and 28-day mortality were then compared between these groups, revealing that the rates of all three of these events were significantly increased among patients with high BAR values relative to those with low BAR values $(P<0.05)$ (Figure 3).

\section{Risk Factors Associated with Sepsis Patient Mortality}

Next, the collected data were subjected to multivariate and univariate binary logistic regression assessments. In univariate assessments, reduced MAP, $\mathrm{SpO}_{2}, \mathrm{pH}, \mathrm{BE}, \mathrm{PLT}$, and ALB values and elevated HR, RR, Lac, BUN, BAR, Cr, PT, and 
Table I Comparison of Baseline Data of Patients with Different Prognosis

\begin{tabular}{|c|c|c|c|c|}
\hline Characteristic & Total Patients $(n=801)$ & Survivors $(n=722)$ & Non-Survivors $(n=79)$ & $\boldsymbol{P}$ \\
\hline \multicolumn{5}{|c|}{ The general information } \\
\hline Age (years) & $57.37 \pm 17.73$ & $57.07 \pm 17.77$ & $60.11 \pm 17.24$ & 0.140 \\
\hline Male, n (\%) & $516(64.4)$ & $467(64.7)$ & $49(62.0)$ & 0.640 \\
\hline $\mathrm{T}\left({ }^{\circ} \mathrm{C}\right)$ & $36.9(36.5,38.0)$ & $36.9(36.6,38.0)$ & $36.7(36.5,37.6)$ & 0.058 \\
\hline HR (time) & $107(95,123)$ & $106(95,122)$ & $114(101,130)$ & 0.002 \\
\hline $\mathrm{RR}$ (time) & $22(21,25)$ & $22(21,24)$ & $24(22,28)$ & $<0.001$ \\
\hline MAP $(\mathrm{mmHg})$ & $89.67(76.50,100.33)$ & $90.00(77.67,100.33)$ & $78.67(68.67,98.67)$ & 0.006 \\
\hline $\mathrm{SpO} 2(\%)$ & $96(92,98)$ & $96(93,98)$ & $94(90,97)$ & 0.005 \\
\hline \multicolumn{5}{|c|}{ Laboratory test results } \\
\hline $\mathrm{PH}$ & $7.44(7.34,7.48)$ & $7.44(7.39,7.48)$ & $7.39(7.30,7.46)$ & $<0.001$ \\
\hline $\mathrm{Lac}(\mathrm{mmol} / \mathrm{L})$ & $1.5(1.2,2.1)$ & I.4 (I.I, 2.0) & $2.6(1.4,2.8)$ & $<0.001$ \\
\hline $\mathrm{BE}(\mathrm{mmol} / \mathrm{L})$ & $22.1(19.3,25.4)$ & $22.55(19.80,25.50)$ & $19.30(15.80,21.30)$ & $<0.001$ \\
\hline WBC $\left(\times 10^{9} / \mathrm{L}\right)$ & II.7| (7.52, I6.33) & II.74 (7.59, I6.29) & II.69 (6.03, I7.03) & 0.672 \\
\hline $\mathrm{HB}(g / L)$ & $111.33 \pm 29.66$ & $113.94 \pm 29.53$ & $108.82 \pm 30.65$ & 0.161 \\
\hline HCT & $0.34(0.29,0.40)$ & $0.34(0.29,0.40)$ & $0.35(0.28,0.39)$ & 0.438 \\
\hline PLT $\left(\times 10^{9} / \mathrm{L}\right)$ & $160(93,240)$ & $163(97,246)$ & $119(55,191)$ & $<0.001$ \\
\hline Bilirubin $(\mu \mathrm{mol} / \mathrm{L})$ & $12.70(8.30,21.90)$ & $12.40(8.30,21.48)$ & $16.00(8.70,23.20)$ & 0.140 \\
\hline ALT (U/L) & $36.0(15.0,55.5)$ & $26.0(16.0,54.0)$ & $29.0(14.0,85.0)$ & 0.514 \\
\hline AST (U/L) & $34.0(22.0,64.5)$ & $33.0(21.0,63.0)$ & $43.0(26.0,125.0)$ & 0.003 \\
\hline ALB $(g / d l)$ & $3.22(2.79,3.72)$ & $3.28(2.82,3.76)$ & $2.84(2.43,3.12)$ & $<0.001$ \\
\hline BUN (mg/dl) & $18.17(12.33,29.37)$ & $17.44(12.06,27.22)$ & $29.29(17.30,49.22)$ & $<0.001$ \\
\hline BAR & $5.67(3.73,9.82)$ & $5.28(3.62,8.75)$ & $10.45(5.90,18.25)$ & $<0.001$ \\
\hline $\mathrm{Cr}(\mu \mathrm{mol} / \mathrm{L})$ & $79.0(60.5,123.0)$ & $77.0(60.0,109.0)$ & I23.0 (74.0, 240.0) & $<0.001$ \\
\hline TG (mmol/L) & I.II $(0.80,1.65)$ & I.II $(0.8 \mathrm{I}, \mathrm{I} .64)$ & I.08 $(0.70,1.68)$ & 0.570 \\
\hline PT (s) & $13.6(12.4,15.4)$ & $13.6(12.4,15.2)$ & $15.4(13.0,18.9)$ & $<0.001$ \\
\hline APTT (s) & $34.1(28.9,40.5)$ & $33.8(28.9,39.7)$ & $38.2(29.7,52.3)$ & $<0.001$ \\
\hline \multicolumn{5}{|l|}{ Scoring system } \\
\hline SOFA (score) & $5(3,8)$ & $4(2,7)$ & $6(9,14)$ & $<0.001$ \\
\hline APACHE II (score) & $15(I I, 20)$ & $15(11,19)$ & $22(17,26)$ & $<0.001$ \\
\hline
\end{tabular}

Abbreviations: T, temperature; HR, heart rate; RR, respiratory rate; MAP, mean arterial pressure; SpO2, oxyhemoglobin saturation; Lac, lactate; $B E$, base excess; WBC, white blood cell; HB, hemoglobin; HCT, haematocrit; PLT, platelet count; ALT, alanine aminotransferase; AST, aspartate aminotransferase; ALB, albumin; BUN, blood urea nitrogen; BAR, blood urea nitrogen-to-albumin ratio; Cr, creatinine; TG, triglyceride; PT, prothrombin time; APTT, activated partial thromboplastin time; SOFA, sequential organ failure assessment; APACHE II, acute physiology and chronic health evaluation II.

APTT values were all identified as risk factors associated with patient 7-day mortality. Following adjustment for age, sex, HR, RR, MAP, SpO2, pH, Lac, BE, PLT, ALB, BUN, Cr, PT, and APTT levels, BAR values were identified as an independent predictor of mortality risk among sepsis patients ( $\mathrm{HR}=1.032,95 \% \mathrm{CI}$ : $1.010-1.055, P=0.004)$. In addition, $\mathrm{RR}, \mathrm{pH}, \mathrm{Lac}, \mathrm{ALB}$, and PT were described as independent predictors of patient mortality. For further details regarding the results of these logistic regression analyses, see Table 2 .

\section{Discussion}

Sepsis and associated multiple organ failure are among the leading causes of global mortality, posing a serious threat to human quality of life. ${ }^{15}$ At present, however, efforts to accurately gauge sepsis severity in affected patients in a timely manner remain difficult. ${ }^{16}$ Several scoring systems and clinical biomarkers have been leveraged for the prognostic evaluation of sepsis patients, but owing to the complex nature of this condition, no specific clinical indicator has been reliably shown to predict outcomes in this patient population. ${ }^{17}$ While the SOFA and APACHE II scores have been employed to estimate sepsis patient mortality in a clinical setting, with higher scores being positively correlated with higher rates of mortality, ${ }^{18-20}$ these scoring systems are time-consuming, complex, and tedious, limiting their more widespread utilization.

BUN levels are commonly employed as a serum biomarker when evaluating renal function, and are routinely utilized in clinical practice owing to the associated ease of measurement. ${ }^{21,22} \mathrm{BUN}$ levels have previously been reported to be 


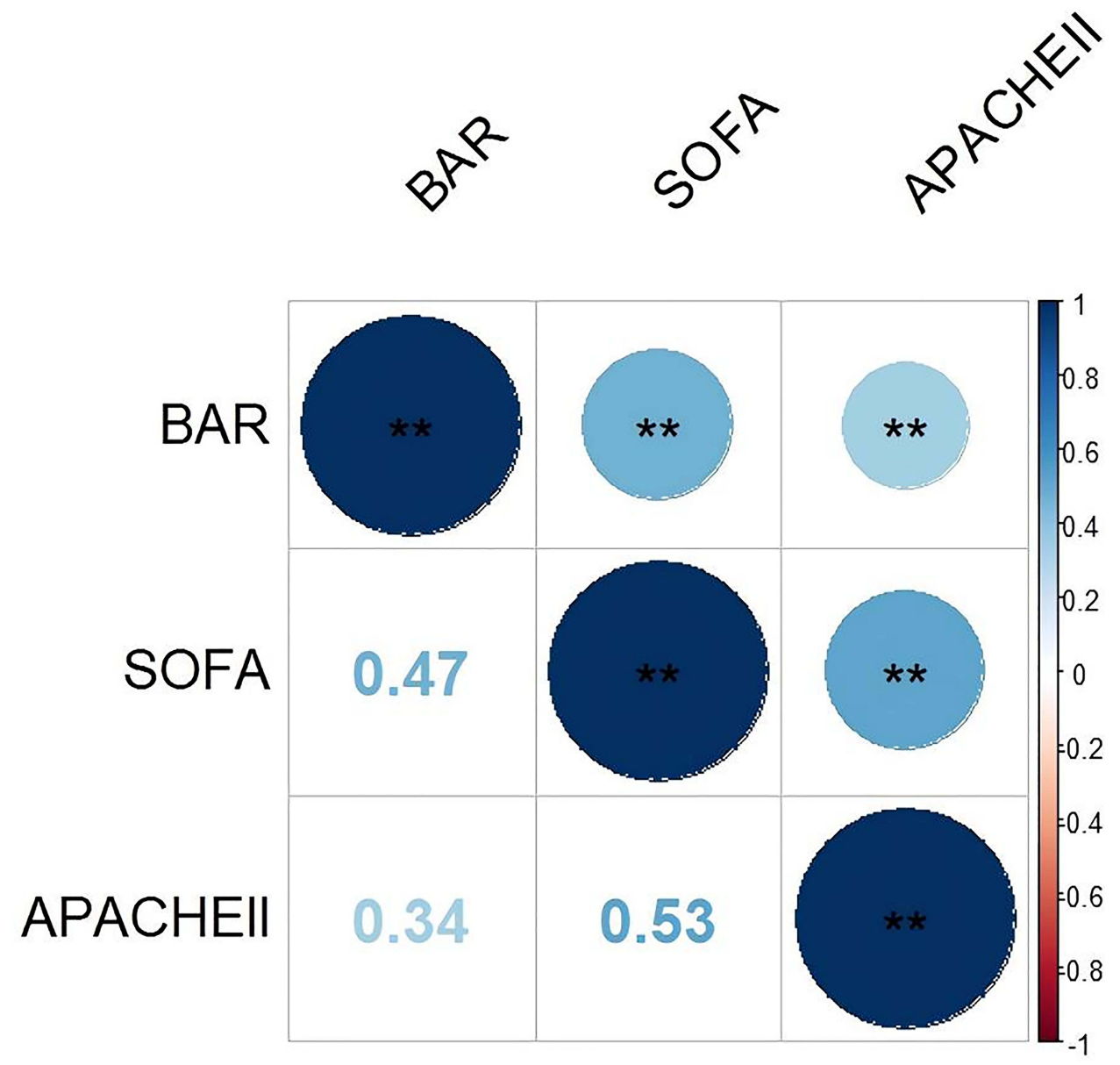

Figure I Correlation coefficient heatmap. $* * P<0.001$.

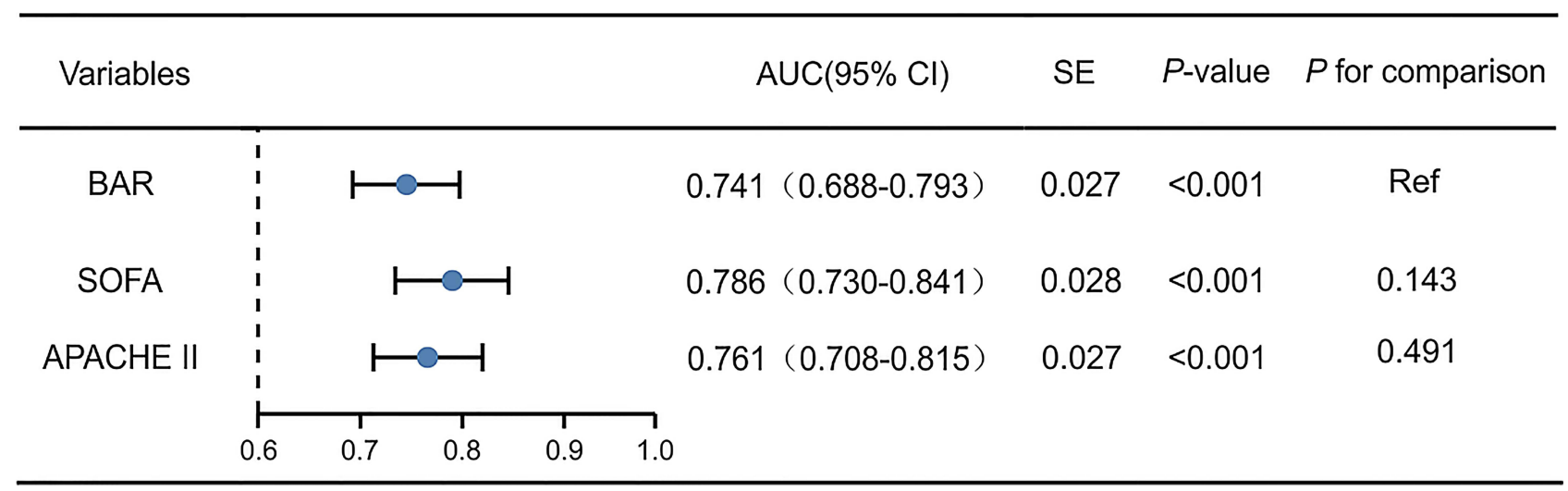

Figure 2 AUC of BAR and other predictive scoring systems for 7-day sepsis mortality.

closely correlated with several diseases including primary aldosteronism (PA), acute pancreatitis (AP), and primary pulmonary hypertension (PPH) and are often used to stratify these patients according to disease severity. ${ }^{23-25}$ BUN levels have also been employed in the context of sepsis patient evaluation in recent years, and have been shown to be a key risk factor associated with poor sepsis patient prognosis. ${ }^{26,27}$ In line with these previous reports, we found that non-surviving sepsis patients exhibited significantly elevated BUN levels as compared to survivors. Further sepsis progression has the potential to result in multiple organ dysfunction, which is the primary reason for mortality among sepsis cases. ${ }^{28}$ The 


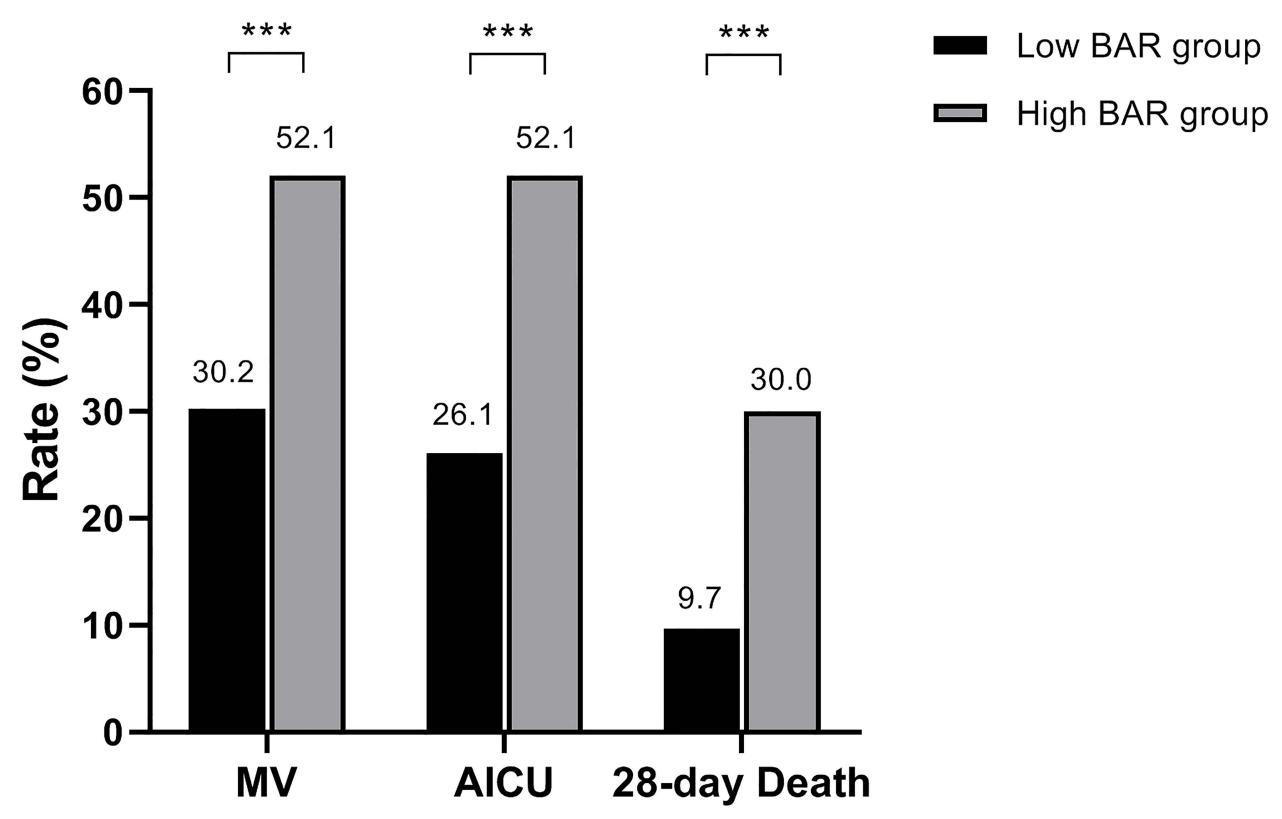

Figure $3 \mathrm{MV}, \mathrm{AICU}$ and 28-day death in the different BAR groups. $* * * P<0.001$.

kidneys are generally the first and most significantly damaged vital in patients suffering from septicemia. ${ }^{29}$ Consistently, BUN levels can serve as an indicator of renal function, rising in the context of renal failure.

Albumin accounts for $\sim 60 \%$ of plasma protein, ${ }^{30}$ playing key physiological roles as a transporter of various compounds, a robust antioxidant, and a regulator of colloidal osmotic pressure within the plasma. ${ }^{31}$ In addition to offering valuable clinical insight regarding patient nutritional status, albumin levels are also associated with acute inflammatory activity in patients. ${ }^{32} \mathrm{~A}$ growing body of evidence has demonstrated the important role played by albumin in the onset and progression of septicemia, underscoring its utility as a prognostic biomarker in the evaluation of septic patients. ${ }^{33,34}$ There are several likely explanations for these findings. For one, severe sepsis can cause systemic inflammation that results in increased vascular permeability and the consequent extravascular leakage of serum albumin, thus resulting in hypoproteinemia outside of the vasculature and causing substantial fluid retention within tissues while simultaneously reducing effective circulating blood volumes, further contributing to the incidence of systemic organ damage. ${ }^{35,36}$ Moreover, the liver is often one of the first and most profoundly affected organs in septicemic patients, and severe damage that exceeds the compensatory capabilities of the liver can compromise albumin synthesis. ${ }^{37}$ Early human serum albumin supplementation has been shown to decrease the incidence of sepsis-related mortality, contributing to better therapeutic achievements in sepsis cases. ${ }^{38}$

Recent articles have highlighted the promise of the BAR as a prognostic inflammation-related biomarker in patients with a range of diseases, but its value in the context of sepsis patient evaluation remains unclear. Here, we therefore explored the utility of BAR values as a predictor of sepsis patient prognosis. This study is the first to our knowledge to have reported increased BAR levels in sepsis patient serum. We also found that serum lactate levels were significantly elevated in patients that did not survive relative to those that did. Higher SOFA and APACHE II scores are generally considered to be indicative of more severe disease and a poorer prognosis. Here, we found that BAR levels were significantly positively correlated with SOFA and APACHE II scores. We then plotted ROC curves and determined the corresponding AUC values as a means of gauging the relative prognostic accuracy of BAR values, SOFA scores, and APACHE II scores when used to evaluate sepsis patients. These analyses revealed that all three approaches were able to effectively establish the risk of 7-day mortality among sepsis patients in the ED. While BAR values performed comparably to SOFA and APACHE II scores, it is important to note that these latter two methods necessitate complex calculations that make their use in an emergency situation impractical. In contrast, BAR values can be readily measured and calculated. To further confirm our findings, cases were separated into two cohorts 
Table 2 Univariable and Multivariable Logistic Regression Analyses of the Various Features Associated with Fatal Outcomes in Patients with Sepsis

\begin{tabular}{|c|c|c|c|c|c|c|c|c|}
\hline \multirow[t]{3}{*}{ Variable } & \multicolumn{4}{|c|}{ Univariable Analysis } & \multicolumn{4}{|c|}{ Multivariable Analysis } \\
\hline & \multirow[t]{2}{*}{ OR } & \multirow[t]{2}{*}{$P$} & \multicolumn{2}{|c|}{ 95\% Cl for $\mathrm{HR}$} & \multirow[t]{2}{*}{ OR } & \multirow[t]{2}{*}{$P$} & \multicolumn{2}{|c|}{ 95\% Cl for $\mathrm{HR}$} \\
\hline & & & Lower & Upper & & & Lower & Upper \\
\hline Age & 1.010 & 0.148 & 0.996 & 1.024 & & & & \\
\hline Gender & 0.892 & 0.640 & 0.552 & 1.440 & & & & \\
\hline $\mathrm{T}$ & 0.921 & 0.278 & 0.793 & 1.069 & & & & \\
\hline HR & 1.017 & 0.004 & 1.005 & 1.028 & & & & \\
\hline $\mathrm{RR}$ & 1.067 & $<0.001$ & 1.031 & 1.104 & 1.064 & 0.002 & 1.024 & 1.107 \\
\hline MAP & 0.983 & 0.009 & 0.970 & 0.996 & & & & \\
\hline $\mathrm{SpO} 2$ & 0.968 & 0.008 & 0.945 & 0.992 & & & & \\
\hline $\mathrm{PH}$ & 0.000 & $<0.001$ & 0.000 & 0.005 & 0.041 & 0.043 & 0.020 & 0.910 \\
\hline Lac & 1.359 & $<0.001$ & 1.235 & 1.495 & 1.261 & $<0.001$ & 1.129 & 1.408 \\
\hline BE & 0.902 & $<0.001$ & 0.864 & 0.942 & & & & \\
\hline WBC & 1.009 & 0.501 & 0.983 & 1.035 & & & & \\
\hline HB & 0.994 & 0.145 & 0.986 & 1.002 & & & & \\
\hline HCT & 0.168 & 0.210 & 0.010 & 2.734 & & & & \\
\hline PLT & 0.996 & 0.001 & 0.993 & 0.998 & & & & \\
\hline Bilirubin & 1.002 & 0.400 & 0.998 & 1.006 & & & & \\
\hline ALT & 1.000 & 0.575 & 1.000 & 1.001 & & & & \\
\hline AST & 1.000 & 0.425 & 1.000 & 1.000 & & & & \\
\hline ALB & 0.325 & $<0.001$ & 0.219 & 0.483 & 0.523 & 0.005 & 0.333 & 0.821 \\
\hline BUN & 1.021 & $<0.001$ & 1.013 & 1.028 & & & & \\
\hline BAR & 1.056 & $<0.001$ & 1.036 & 1.076 & 1.032 & 0.004 & 1.010 & 1.055 \\
\hline $\mathrm{Cr}$ & 1.002 & 0.003 & 1.001 & 1.002 & & & & \\
\hline TG & 0.963 & 0.677 & 0.808 & 1.149 & & & & \\
\hline PT & 1.053 & $<0.001$ & 1.026 & 1.081 & 1.031 & 0.005 & 1.010 & 1.054 \\
\hline APTT & $\mathrm{I} .027$ & $<0.001$ & 1.016 & 1.038 & & & & \\
\hline
\end{tabular}

Notes: Adjusted for age, sex, HR, RR, MAP, SpO 2 , PH, Lac, BE, PLT, ALB, BUN, Cr, PT and APTT.

Abbreviations: T, temperature; HR, heart rate; RR, respiratory rate; MAP, mean arterial pressure; SpO2, oxyhemoglobin saturation; Lac, lactate; $B E$, base excess; WBC, white blood cell; HB, hemoglobin; HCT, haematocrit; PLT, platelet count; ALT, alanine aminotransferase; AST, aspartate aminotransferase; ALB, albumin; BUN, blood urea nitrogen; BAR, blood urea nitrogen-to-albumin ratio; $\mathrm{Cr}$, creatinine; TG, triglyceride; PT, prothrombin time; APTT, activated partial thromboplastin time.

according to their BAR values. Further analyses exhibited that there were substantial discrepancies between these two groups with respect to secondary endpoints including AICU, MV, and 28-day mortality. As such, BAR values can be readily leveraged to monitor therapeutic responses and/or deterioration in sepsis patients in the ED.

\section{Limitations}

There are certain limitations to these analyses. For one, this was a retrospective single-center research with a small sample size, potentially subjecting these achievements to selection bias. Therefore, the analysis does not adjust for potential confounders. Although the sample size was relatively small, according to the sample size calculations the minimum number required for each group was met. Second, we did not conduct any external validation, and these results thus ought to be validated in the next large-scale investigations. Lastly, it is possible that dynamic changes in BAR values may offer greater prognostic value for the evaluation of sepsis patients, but we failed to test this possibility in the present study.

\section{Conclusion}

In conclusion, BAR value at admission is an easily measured biomarker that is positively correlated with sepsis severity, with a higher BAR value being indicative of a poorer sepsis patient prognosis. 


\section{Acknowledgment}

We are extremely grateful to all the volunteers who took part in this study and all the participants for their contribution to data collection and analysis.

\section{Funding}

This work was supported by:1. Key R\&D Project of Sichuan Provincial Department of Science and Technology (2021YFS0023).2. Technology Innovation Project of Key R \& D Support Plans of Chengdu Science and Technology Municipality (2020-YF05-00074-SN).3. Project of the Beijing Medical and Health Foundation (YWJKJJHKYJJB184096-Q26).

\section{Disclosure}

The authors have no potential conflicts of interest to disclose.

\section{References}

1. Wu M-H, Tsou P-Y, Wang Y-H, et al. Impact of post-sepsis cardiovascular complications on mortality in sepsis survivors: a population-based study. Critical Care. 2019;23(1):293. doi:10.1186/s13054-019-2579-2

2. Fleischmann C, Scherag A, Adhikari NKJ, et al. Assessment of global incidence and mortality of hospital-treated sepsis. current estimates and limitations. Am J Respir Crit Care Med. 2016;193(3):259-272. doi:10.1164/rccm.201504-0781oc

3. Han X, Edelson DP, Snyder A, et al. Implications of Centers for medicare \& medicaid services severe sepsis and septic shock early management bundle and initial lactate measurement on the management of sepsis. Chest. 2018;154(2):302-308. doi:10.1016/j.chest.2018.03.025

4. Singer M, Deutschman CS, Seymour CW, et al. The third international consensus definitions for sepsis and septic shock (Sepsis-3). JAMA. 2016;315(8):801-810.

5. Schutte K, Malfertheiner P. Markers for predicting severity and progression of acute pancreatitis. Best Pract Res Clin Gastroenterol. 2008;22 (1):75-90. doi:10.1016/j.bpg.2007.10.013

6. Coopersmith CM, De Backer D, Deutschman CS, et al. Surviving sepsis campaign: research priorities for sepsis and septic shock. Intensive Care Med. 2018;44(9):1400-1426. doi:10.1007/s00134-018-5175-z

7. Donnelly JP, Safford MM, Shapiro NI, Baddley JW, Wang HE. Application of the third international consensus definitions for sepsis (Sepsis-3) Classification: a retrospective population-based cohort study. Lancet Infect Dis. 2017;17(6):661-670. doi:10.1016/S1473-3099(17)30117-2

8. Zhao X-J, Li Q-X, Chang L-S, et al. Evaluation of the Application of APACHE II Combined With NIHSS Score in the Short-Term Prognosis of Acute Cerebral Hemorrhage Patient. Front Neurol. 2019;10:475. doi:10.3389/fneur.2019.00475

9. Li D, Zhou Y, Yu J, et al. Evaluation of a novel prognostic score based on thrombosis and inflammation in patients with sepsis: a retrospective cohort study. Clin Chem Lab Med. 2018;56(7):1182-1192. doi:10.1515/cclm-2017-0863

10. Dundar ZD, Kucukceran K, Ayranci MK. Blood urea nitrogen to albumin ratio is a predictor of in-hospital mortality in older emergency department patients. Am J Emerg Med. 2021;46:349-354. doi:10.1016/j.ajem.2020.10.008

11. Huang D, Yang H, Yu H, et al. Blood Urea Nitrogen to Serum Albumin Ratio (BAR) Predicts Critical Illness in Patients with Coronavirus Disease 2019 (COVID-19). Int J Gen Med. 2021;14:4711-4721. doi:10.2147/JJGM.S326204

12. Küçükceran K, Ayrancı MK, Girişgin AS, Koçak S, Dündar ZD. The role of the BUN/albumin ratio in predicting mortality in COVID-19 patients in the emergency department. Am J Emerg Med. 2021;48:33-37. doi:10.1016/j.ajem.2021.03.090

13. Bae SJ, Lee SH, Yun SJ, Kim K. Comparison of IVC diameter ratio, BUN/creatinine ratio and BUN/albumin ratio for risk prediction in emergency department patients. Am J Emerg Med. 2021;47:198-204. doi:10.1016/j.ajem.2021.03.081

14. Liu B, Li D, Cheng Y, et al. Development and internal validation of a simple prognostic score for early sepsis risk stratification in the emergency department. BMJ open. 2021;11(7):e046009-e046009. doi:10.1136/bmjopen-2020-046009

15. Robert Boter N, Mòdol Deltell JM, Casas Garcia I, Rocamora Blanch G, Lladós Beltran G, Carreres Molas A. Activation of code sepsis in the emergency department is associated with a decrease in mortality. Medicina Clinica. 2019;152(7):255-260. doi:10.1016/j.medcle.2019.01.013

16. Charles PE, Noel R, Massin F, et al. Significance of soluble triggering receptor expressed on myeloid cells-1 elevation in patients admitted to the intensive care unit with sepsis. BMC Infect Dis. 2016;16(1):559. doi:10.1186/s12879-016-1893-4

17. Jiang W, Li X, Wen M, et al. Increased percentage of PD-L1(+) natural killer cells predicts poor prognosis in sepsis patients: a prospective observational cohort study. Critical Care. 2020;24(1):617. doi:10.1186/s13054-020-03329-z

18. Anami EHT, Grion CMC, Cardoso LTQ, et al. Serial evaluation of SOFA score in a Brazilian teaching hospital. Intensive Critical Care Nursing. 2010;26(2):75-82. doi:10.1016/j.iccn.2009.10.005

19. Krasselt M, Baerwald C, Petros S, Seifert O. Outcome of Patients With Necrotizing Vasculitis Admitted to the Intensive Care Unit (ICU) for Sepsis: results of a Single-Centre Retrospective Analysis. J Intensive Care Med. 2021;36(12):1410-1416. doi:10.1177/0885066620953768

20. Sadaka F, EthmaneAbouElMaali C, Cytron MA, Fowler K, Javaux VM, O'Brien J. Predicting Mortality of Patients With Sepsis: a Comparison of APACHE II and APACHE III Scoring Systems. J Clin Med Res. 2017;9(11):907-910. doi:10.14740/jocmr3083w

21. Wang X, Liang H, Wang Y, et al. Risk factors of renal dysfunction and their interaction in level-low lead exposure paint workers. BMC Public Health. 2018;18(1):526. doi:10.1186/s12889-018-5475-9

22. Li L, Chen Y, Jiao D, Yang S, Li L, Li P. Protective effect of astaxanthin on ochratoxin a-induced kidney injury to mice by regulating oxidative stress-related NRF2/KEAP1 Pathway. Molecules. 2020;25(6):1386. doi:10.3390/molecules25061386

23. Liu Y, Zhou L, Liu Z, et al. Higher Blood urea nitrogen and urinary calcium: new risk factors for diabetes mellitus in primary aldosteronism patients. Front Endocrinol (Lausanne). 2020;11:23. doi:10.3389/fendo.2020.00023 
24. Hu B, Xu G, Jin X, et al. Novel prognostic predictor for primary pulmonary hypertension: focus on blood urea nitrogen. Front Cardiovascular Med. 2021;8:724179. doi:10.3389/fcvm.2021.724179

25. Pando E, Alberti P, Mata R, et al. Early Changes in Blood Urea Nitrogen (BUN) Can Predict Mortality in Acute Pancreatitis: comparative Study between BISAP Score, APACHE-II, and Other Laboratory Markers-A Prospective Observational Study. Canadian $j$ Gastroenterol Hepatol. 2021;2021:6643595. doi:10.1155/2021/6643595

26. Li X, Li T, Wang J, et al. Higher blood urea nitrogen level is independently linked with the presence and severity of neonatal sepsis. Ann Med. 2021;53(1):2192-2198. doi:10.1080/07853890.2021.2004317

27. Li X, Zheng R, Zhang T, Zeng Z, Li H, Liu J. Association between blood urea nitrogen and 30-day mortality in patients with sepsis: a retrospective analysis. Ann Palliat Med. 2021;10(11):11653-11663. doi:10.21037/apm-21-2937

28. Demoule A, Jung B, Prodanovic H, et al. Diaphragm Dysfunction on Admission to the Intensive Care Unit. Prevalence, Risk Factors, and Prognostic Impact-A Prospective Study. Am J Respir Crit Care Med. 2013;188(2):213-219. doi:10.1164/rccm.201209-1668oc

29. Schrier RW, Wang W. Acute Renal Failure and Sepsis. N Eng J Med. 2004;351(2):159-169. doi:10.1056/nejmra032401

30. Ikeda M, Ishima Y, Chuang VTG, et al. Distribution of Polysulfide in Human Biological Fluids and Their Association with Amylase and Sperm Activities. Molecules. 2019;24(9):1689. doi:10.3390/molecules24091689

31. Choi JW, Park J-S, Lee CH. Genetically determined hypoalbuminemia as a risk factor for hypertension: instrumental variable analysis. Sci Rep. 2021;11(1):11290. doi:10.1038/s41598-021-89775-3

32. Li M, Tang C, Luo E, Qin Y, Wang D, Yan G. Relation of Fibrinogen-to-Albumin Ratio to Severity of Coronary Artery Disease and Long-Term Prognosis in Patients with Non-ST Elevation Acute Coronary Syndrome. Biomed Res Int. 2020;2020:1860268. doi:10.1155/2020/1860268

33. Yoon SH, Shin H, Lee KH, et al. Predictive factors for bacteremia in febrile infants with urinary tract infection. Sci Rep. 2020;10(1):4469. doi:10.1038/s41598-020-61421-4

34. Xiao G, Liao W, Zhang Y, et al. Analysis of fungal bloodstream infection in intensive care units in the Meizhou region of China: species distribution and resistance and the risk factors for patient mortality. BMC Infect Dis. 2020;20(1):599. doi:10.1186/s12879-020-05291-1

35. Ohta Y, Miyamoto K, Kawazoe Y, Yamamura H, Morimoto T. Effect of dexmedetomidine on inflammation in patients with sepsis requiring mechanical ventilation: a sub-analysis of a multicenter randomized clinical trial. Critical Care. 2020;24(1):493. doi:10.1186/s13054-020-03207-8

36. Shekar K, Roberts JA, Barnett AG, et al. Can physicochemical properties of antimicrobials be used to predict their pharmacokinetics during extracorporeal membrane oxygenation? Illustrative data from ovine models. Critical Care. 2015;19:437. doi:10.1186/s13054-015-1151-y

37. Grau T, Bonet A, Rubio M, et al. Liver dysfunction associated with artificial nutrition in critically ill patients. Critical Care. 2007;11(1):1-12.

38. Philips CA, Maiwall R, Sharma MK, et al. Comparison of 5\% human albumin and normal saline for fluid resuscitation in sepsis induced hypotension among patients with cirrhosis (FRISC study): a randomized controlled trial. Hepatol Int. 2021;15(4):983-994. doi:10.1007/s12072021-10164-z

Journal of Inflammation Research

Dovepress

\section{Publish your work in this journal}

The Journal of Inflammation Research is an international, peer-reviewed open-access journal that welcomes laboratory and clinical findings on the molecular basis, cell biology and pharmacology of inflammation including original research, reviews, symposium reports, hypothesis formation and commentaries on: acute/chronic inflammation; mediators of inflammation; cellular processes; molecular mechanisms; pharmacology and novel anti-inflammatory drugs; clinical conditions involving inflammation. The manuscript management system is completely online and includes a very quick and fair peer-review system. Visit http://www.dovepress.com/testimonials.php to read real quotes from published authors.

Submit your manuscript here: https://www.dovepress.com/journal-of-inflammation-research-journal 